\title{
Questes
}

Revue pluridisciplinaire d'études médiévales

$13 \mid 2008$

Figures royales à l'ombre du mythe

\section{Quand l'ours devient faible ou Arthur et ses défaillances}

Pierre Levron

\section{(2) OpenEdition}

1 Journals

Édition électronique

URL : http://journals.openedition.org/questes/1487

DOI : 10.4000/questes. 1487

ISSN : 2109-9472

Éditeur

Les Amis de Questes

\section{Édition imprimée}

Date de publication : 15 janvier 2008

Pagination : 8-23

ISSN : 2102-7188

\section{Référence électronique}

Pierre Levron, «Quand l'ours devient faible ou Arthur et ses défaillances », Questes [En ligne], 13 | 2008, mis en ligne le 01 janvier 2014, consulté le 25 septembre 2020. URL : http://journals.openedition.org/ questes/1487; DOI : https://doi.org/10.4000/questes.1487

\section{(C) Association des amis de «Questes »}




\section{Quand l'ours devient faible ${ }^{1}$ \\ ou \\ Arthur et ses défaillances}

\section{Pierre LEVRON}

Exemple essentiel de souverain mythique, par les origines qu'on lui attribue aussi bien que par les qualités que Robert Wace lui reconnaît dans le $B r u t^{2}$, Arthur est l'une des figures capitales de la royauté littéraire. S'il parvient à écarter les envahisseurs du royaume de Logres et à instaurer une paix durable, ce roi puissant n'est pas exemplaire par ses seules vertus : ses défaillances, assez fréquentes dans les romans de la «matière de Bretagne» contribuent à une confrontation du rôle référentiel que l'on prête au personnage avec la fragilité qui caractérise parfois l'homme. Que sont les défaillances d'Arthur? Ce sont surtout des crises psychologiques ou éthiques qui concernent l'individu et qui mettent parfois très sérieusement en cause sa capacité à exercer sa fonction et à satisfaire aux impératifs symboliques et moraux supposés par l'institution royale. Les défaillances d'Arthur sont toujours publiques : elles l'opposent à un (ou plusieurs) personnages qui lui font des remontrances et cherchent à susciter une réaction positive de sa part. Elles ont une cause majeure : la mélancolie du roi $^{3}$, et concernent ses fonctions militaires et chevaleresques, ses prérogatives judiciaires et son rôle religieux; elles servent à définir l'humanité d'un personnage investi d'une fonction référentielle et à interroger son comportement

\footnotetext{
${ }^{1}$ Pour les liens étymologiques et symboliques entre Arthur et l'ours, voir Philippe WALTER, Arthur, l'ours et le roi, Paris, IMAGO, 2002.

${ }^{2}$ Voir Dominique Boutet, Charlemagne et Arthur, ou le roi imaginaire, Paris, Champion, 1992 (coll. Nouvelle bibliothèque du Moyen Âge).

${ }^{3}$ Voir Pierre LEVRon, Naissance de la mélancolie dans la littérature des douzièmes et treizièmes siècles, thèse de doctorat dirigée par Jacqueline Cerquiglini-Toulet et soutenue le 30 juin 2005 à l'université de Paris-Sorbonne (Paris-IV).
} 
vis-à-vis des aspects surnaturels de sa charge. Après avoir défini les caractéristiques de la mélancolie d'Arthur, nous examinerons ses défaillances amoureuses et les crises de la souveraineté, en interrogeant des textes acclimatant la légende arthurienne dans les langues vernaculaires comme le Conte du Graal ou Jaufre, que des romans en prose qui amplifient les récits sur Arthur et sa cour en révélant parfois ce que leurs devanciers taisent.

\section{Arthur, roi mélancolique}

La mélancolie du roi ne préoccupe pas seulement les écrivains de la fin du Moyen $\hat{A} g e^{4}$; leurs devanciers du XII ${ }^{\mathrm{e}}$ et XIII ${ }^{\mathrm{e}}$ siècles s'avèrent y être très sensibles $^{5}$. Cette mélancolie est une passion inquiétante, inspirée du concept théologique d'acedia aussi bien que des théories médicales hippocratiques et galéniques décrivant la chimie des humeurs. Les romanciers la définissent souvent par la tristesse et pratiquement toujours par l'incapacité à remplir une fonction sociale ou à satisfaire une vocation importante pour la société. C'est l'un des fondements capitaux d'une «mélancolie arthurienne »: devenu mélancolique, le roi n'est plus capable de remplir son rôle référentiel. Sa mélancolie est un repli psychologique sur lui-même qui le conduit à s'abstraire du monde environnant. Elle possède deux espèces importantes : la « diversion mélancolique » lors de laquelle Arthur s’abîme publiquement dans ses pensées (c'est donc une «mélancolie interne ») et la «mélancolie conflictuelle » où le conflit entre ce qu'il ressent et ce qu'il devrait faire est beaucoup plus vif, le personnage agissant à rebours de ce que l'on attend de lui. La « diversion

\footnotetext{
4 Voir Joël BLANCHARD, «Le corps du roi : mélancolie et 'recreation', implications médicales et culturelles du loisir des princes à la fin du Moyen Âge», Représentation, pouvoir et royauté à la fin du Moyen Âge, édité par Joël BlANCHARD, postface de Philippe CONTAMINE, Paris, Picard, 1995, p. 139-211.

${ }^{5}$ Bien que la démarche des écrivains soit très différente de celle d'un clerc comme Gervais de Tilbury composant ses Otia Imperalia pour Otton IV de Brunswick (voir la traduction d'Annie Duchesne, préface de Jacques Le GofF, Paris, Les Belles-Lettres, 1992, coll. La Roue à livres), leurs préoccupations se rejoignent : la mélancolie royale (défaillance morale ou soucis accablants) doit être combattue.
} 
mélancolique » est fréquente dans les romans bretons du Moyen Âge central. Elle survient en général pendant une période brève, comme le montre ce passage du Lancelot en Prose :

« Au chief de trois jours avint que li rois seoit au disner. Et quant il ot une piece mengié, si comança a penser mout durement; et bien paroit a son penser que ses cuers n'estoit mie a ese, ançois deïst bien (qui le veïst) que mout ert a malaise $»^{6}$.

La diversion mélancolique est toujours un spectacle; elle se produit ici pendant un repas. Elle rompt avec la logique commune (la commensalité courtoise) par l'isolement d'Arthur dans une pensée obsessionnelle rendue très visible par son attitude : un état divergent doit toujours matérialiser la souffrance mélancolique du personnage. Les écrivains insistent beaucoup sur cet aspect. Il arrive ainsi que des péripéties enrichissent l'attitude du roi. Arthur peut pleurer ${ }^{7}$, ou casser la lame du couteau qu'il tient en s'appuyant dessus ${ }^{8}$. L'auteur de la Première continuation de Perceval ${ }^{9}$ le montre se blessant avec un autre de ces ustensiles. L'idée d'une pensée obsédante pathologique, qui contribue à définir l'amour hérö̈que chez Bernard de Gordon ${ }^{10}$, est réalisée ici par la substitution d'une activité inconsciente à des comportements idéalement gouvernés par la conscience. Le traumatisme physique est parfois remplacé par la distance (physique) que prend Arthur par rapport à ses vassaux. Le même roman le montre ainsi en train de prendre à deux reprises du retard sur plusieurs

\footnotetext{
${ }^{6}$ Lancelot $d u$ Lac, édité par Elspeth KENNEDY, traduit et présenté par François MoSES, Paris, Librairie Générale Française, 1991 (coll. Le livre de poche. Lettres Gothiques), tome I, chapitre L, p. 778.

${ }^{7}$ Ibid., chapitre XIX, p. 386.

${ }^{8}$ Voir L'Atre Périlleux, édité par Brian Woledge, Paris, Champion, 1936 (coll. CFMA), vers 298-305.

9 Éditée par Colette-Anne VAn COOLPUT-STORMS, Paris, Librairie Générale Française, 1993 (coll. Le livre de poche. Lettres gothiques), vers 3414-3425.

${ }^{10}$ Bernard de Gordon, Lilium Medicinae (particula II), in John Livingston Lowes, «The loveres maladye of hereos », Modern Philology, 11 (1914), p. 491-546 et notamment p. 497502 ; Giorgio AGAMBEN, Stanze, parole et fantasme dans la culture occidentale, Paris, Payot, 1994, p. 184-206.
} 
chevaliers qui l'accompagnent à la chasse ${ }^{11}$. Dans ce cas, le roi quitte l'ensemble cohérent qu'il forme avec sa suite. Le romancier joue subtilement avec la grande ambiguïté de la chasse dans la littérature médiévale : si elle contribue à prévenir les états mélancoliques parce qu'elle impose une hygiène de vie très rigoureuse et parce qu'elle est un très grand plaisir social et sportif ${ }^{12}$, elle peut également les favoriser (ou les illustrer) ${ }^{13}$. Les diversions mélancoliques sont toujours implicitement perçues comme des défaillances morales chez un personnage qui doit être le garant aussi bien de la joie de sa cour que de la cohésion de son réseau vassalique. Un état divergent est donc envisagé simultanément du point de vue des tenants de la norme sociale et de celui d'Arthur. L'arrivée de Perceval à sa cour dans le Conte du Graal donne lieu à deux appréhensions dans la tradition manuscrite du roman: la rédaction du manuscrit Paris, BNF fr. 12576 oppose simplement la joie des chevaliers au mutisme pensif du roi $^{14}$ alors que celle du manuscrit Berne 354 décrit la perplexité des vassaux d'Arthur contemplant leur suzerain ${ }^{15}$. Le regard du narrateur va donc de la mesure objective en apparence d'une tension entre la mélancolie du souverain et les plaisanteries des courtisans à l'interrogation. Dans les deux cas, il oppose une norme que le roi ne garantit ni n'incarne momentanément plus à une posture mélancolique soulignée par une coupure logique (l'attitude du roi est l'inverse de celle de ses commensaux) ou par un scandale formulé par une question soulignant la rupture d'une continuité normative entre le souverain et ses

\footnotetext{
${ }^{11}$ Première Continuation de Perceval, vers $2130-2133$ et 3291-3298.

12 Voir La Diététique à Alexandre, éditée par Hermann SUCHIER, Denkmäler der provenzalischer Literatur und Sprache, Halle, Max Niemeyer, 1883, tome I, p. 201-213, vers 242 ; Armand Strubel, Chantal De Saulnier, La Poétique de la chasse au Moyen Âge, Paris, PUF, 1994 (coll. Perspectives littéraires).

${ }^{13}$ Voir le Dit du cerf amoureux, édité par Bernard CHARRIER, Perspectives Médiévales, 12 (1986), p.5-12. La chasse inspire un récit métaphorique de poursuite amoureuse obsessionnelle.

${ }^{14}$ Chrétien De Troyes, Le Conte du Graal, édité par William RoACH, Genève, Droz / Paris, Mignard, 1959 (coll. TLF, 71), vers 907-911.

${ }^{15}$ Rédaction éditée par Charles MÉLA, Paris, Librairie Générale Française, 1992 (coll. Le livre de poche. Lettres Gothiques), vers 865-869.
} 
vassaux. L'auteur de Floriant et Florete ${ }^{16}$ préfère décrire l'unisson de la tristesse collective et de la mélancolie du roi. Manquant à sa fonction sociale, Arthur se comporte dans tous les cas à l'encontre de l'exigence aristocratique de sociabilité joyeuse. L'association de la tristesse du roi à la mélancolie se fait, par-delà l'influence de l'acedia, par une tension entre la tristesse atrabilaire et l'idéal humoral et social représenté par un tempérament sanguin vecteur de qualités appréciées par les nobles, comme la gaietét ${ }^{17}$.

Les mélancolies « externes » sont beaucoup plus sensibles à ce risque de contagion atrabilaire. Elles consistent parfois en la prolongation d'une tristesse profonde, comme dans l'Estoire de Merlin ${ }^{18}$. Reprenant le schéma narratif d'une diversion mélancolique, le récit en dilate la durée et l'enrichit d'une attente inutile. La crise se manifeste par la fixation psychique et affective d'Arthur dans une posture qui contredit le dynamisme général d'une narration montrant un jeune roi accéder à la souveraineté. Dans le Perlesvaus, Arthur cesse de réunir sa cour :

« Li roiz Artus aprés la mort son pere mena la plus haute vie et la plus cointe que nus rois menast onques, si que tui(t) li prince et li baron prenoient essanple a lui de bien fere. Li rois Artuz fu .X. anz en tel part com ge vos di, ne n'estoit nus rois heureus et tant liez comme lui, tant que une volentez deloiauz li vint, e commença a perdre le talent as largesces que il soloit fere. Ne voloit cort tenir a Noël, ne a Pasques, ne a Pentecoste. Li chevalier de la Table Reonde, qant il virent son bienfait alentir, il s'en partirent e commencierent sa cort a lessier. De trois .C. et .LXX. chevaliers qu'il soloit avoir de sa mesniee, n'avoit il ore mie plus de.XXV. au plus. Nulle aventure n'avenoit mie en sa cort. Tuit li autre prince

\footnotetext{
${ }^{16}$ Édité par Annie COMBES et Richard TrAChSLER, Paris, Champion, 2003 (coll. Champion classiques : Moyen Âge), vers 1185-1191.

${ }^{17}$ Voir le Dialogue de Placides et Timéo, édité par Claude Thomasset, Genève, Droz, 1980 (coll. TLF, 289).

${ }^{18}$ L'Estoire de Merlin (Les premiers faits du roi Arthur), édité par Irène FrEIRE-NuNES, traduit et annoté par Anne BERTHELOT et Philippe WALTER, Le livre du Graal, tome I, Paris, Gallimard, 2002 (coll. La Pléiade), p. 809-1662.
} 
avoient leur bienfait deloez por ce qu'il veoient le roi maintenir si foiblement $\gg{ }^{19}$.

Passant d'un rôle référentiel à une posture marginale, Arthur éprouve une mélancolie illogique en apparence, puisque le narrateur décrit son aboutissement mais non sa cause fondamentale : la défaillance de Perlesvaus qui n'a pas su poser la question permettant au Roi-Pécheur de guérir. Le roman oppose un schéma narratif de clinique littéraire (c'est-à-dire de guérison d'une crise atrabilaire) fondé sur une prise de conscience ${ }^{20}$ à une rupture de prime abord brutale et totale d'une vocation référentielle. Il décrit donc un véritable « arbitraire mélancolique » fondé sur un conflit entre les mouvements de l'esprit du roi et la norme qu'il commence par incarner. Cette rupture est d'autant plus violente qu'il est impossible de reconstituer l'évolution intellectuelle du personnage. Le roman décrit une contagion mélancolique concernant l'ensemble de son réseau vassalique et de la société nobiliaire, la suspension des aventures allant de pair avec la disparition de la générosité chez les nobles. Devenue norme sociale, la mélancolie n'est plus scandaleuse que pour quelques personnages pourvus d'une autorité morale comme Guenièvre ou une demoiselle qui dénoncera la «malvaistié » du roi, dès lors que les vassaux ou les compagnons du roi ne le rappellent plus à l'ordre . La mélancolie « externe » est donc définie par deux critères essentiels : un violent conflit logique entre le fonctionnement intime du roi et les circonstances extérieures, ainsi que son caractère presque toujours contagieux. La sensibilité d'Arthur à la mélancolie permet de décrire un personnage particulièrement sensible aux failles de son psychisme. Le roi n'est pas moins exposé à une passion qui se rapproche de la mélancolie par son caractère impérieux, l'amour, qui est lui aussi une cause importante de défaillances.

${ }^{19}$ Le Haut livre du Graal, édité par Armand STRUBEL, Paris, Librairie Générale Française, 2007 (coll. Le Livre de poche. Lettres Gothiques), branche I, p. 132.

${ }^{20}$ Ibid., p. 142- 174 : sur le conseil de Guenièvre, Arthur se rend à la chapelle de SaintAugustin qui est dans la Blanche Forêt. 


\section{L'amour et ses défaillances}

Les écrivains du Moyen Âge central traitent souvent des amours du roi Arthur en opposant son mariage légitime avec Guenièvre à des passions dangereuses, qui mettent gravement en cause sa capacité à exercer la royauté. L'amour incestueux d'Arthur pour sa demi-sœur et sa passion pour la fausse Guenièvre sont extrêmement caractéristiques des défaillances amoureuses du roi, puisqu'ils révèlent des crises morales profondes. La conception de Mordret est le fruit d'un inceste ; elle est relatée par les suites Vulgate et Post-Vulgate du Roman de Merlin. La Suite du Roman de Merlin ${ }^{21}$ commence par souligner que l'épouse du roi Loth ignore qu'Arthur est son frère : " Mais quoi que elle fust sa suer n'en savoit elle riens $»^{22}$. L'auteur de l'Estoire de Merlin le souligne également, mais adopte une stratégie narrative très différente: la passion amoureuse (pour la dame) est relatée sans allusion à leurs liens familiaux ${ }^{23}$. Ensuite, le jeune homme se substitue à Loth :

«Ensi s'en ala li rois au parlement a la Crois et la dame remest toute seul illuec gisant. Et Artus, qui bien s'en estoit pris garde, ot bien veü conment li rois s'en fu partis. Si se leva et ala u lit a la dame et se coucha avoec li. Et quant il fu couchiés si se torna et retorna que autre chose n'en osa faire. Et il avint chose que la dame s'esveilla et se tourna devers li come feme endormie, si quida vraiement que ce fust ses sires, si l'embracha, et quant il fu embraciés si sot bien qu'ele ne se prenoit garde de lui, si l'embracha. Et jut o lui tout plainnement. Si li fist la dame molt grant joie et bien li fist, car elle quida que ce fust ses sires. Et en tel manière fu Mordrés engendrés $»^{24}$.

L'insinuation d'Arthur dans le lit de la dame et surtout l'instauration d'une duplicité essentielle entre une union charnelle effective et une union matrimoniale illusoire rendent possible la substitution de l'inceste à des relations

\footnotetext{
${ }^{21}$ Éditée par Gilles Roussineau, Genève, Droz, 2006 (coll. TLF, 972).

${ }^{22}$ La Suite du Roman de Merlin, op. cit, chapitre I, paragraphe 1, p. 1.

${ }^{23}$ L'Estoire de Merlin (les premiers faits du roi Arthur), op. cit, chapitre 58, p. 869.

${ }^{24}$ Ibid., paragraphe 59, p. 870.
} 
sexuelles légitimées par le mariage et par la conception d'un héritier. Avant d'être qualifié formellement d'inceste ${ }^{25}$, son commerce charnel avec sa sœur s'apparente à un adultère par substitution astucieuse qui se différencie de la conception d'Arthur par Uterpendragon par l'absence totale de médiateur : le jeune homme agit totalement de lui-même. Événement unique, l'inceste témoignera donc de l'immaturité fondamentale d'un personnage caractérisé d'ailleurs par plusieurs traits idéaux de l'adolescent noble : sa beauté et sa joie de vivre $^{26}$. En utilisant des caractéristiques destinées ouvertement à le rendre aimable, le narrateur brouille son identité : Arthur n'est visuellement pas le frère de la dame, mais un jeune homme qu'elle ne peut distinguer des autres ni dominer. Les auteurs jouent sur l'ambiguité fondamentale d'une situation où le frère et la sœur sont étrangers l'un à l'autre : le désir amoureux éprouvé par Arthur s'oppose donc à ce que les destinataires du récit savent de lui et de la dame. S'il s'agit d'un inceste «objectif» au sens où il est formellement constitué sans que les partenaires en soient conscients, il n'en reste pas moins vrai que la défaillance du roi repose, dans les deux textes, sur la concomitance de la transgression d'un interdit civilisateur et de la soumission à un désir sexuel qu'il ne parvient pas à vaincre. La conception de Mordret est un exemple de confrontation entre la pulsion amoureuse et les normes collectives; elle est donc un avatar néfaste de l'exception essentielle d'un héros fondateur, en même temps qu'une réécriture spéculaire de la conception d'Arthur. Elle en reprend une donnée importante : le caractère adultère d'une union où Arthur se substitue physiquement à Loth jusqu'à se confondre avec lui (dans la Suite-Vulgate) ou à chercher à retenir son aimée à l'instar de son père dans le Roman de Merlin

\footnotetext{
${ }^{25}$ Ibid., p. 871 : «Et ensi jut Artus o sa serour, mais onques puis ne li avint, si sot bien la dame qu'ele fu grosse de lui $»$.

${ }^{26}$ Voir L'Estoire de Merlin (les premiers faits du roi Arthur), op. cit, paragraphe 58, p. 869 : «Et Artus estoit molt biaus vallés et molt envoisiés ». On comparera avec d'autres portraits d'adolescents nobles, et en particulier celui de Sone de Nansay, qui présente lui aussi ces traits. Voir, ici, Claude LACHET, Sone de Nansay et le roman d'aventure en vers au treizième siècle, Paris, Champion, 1992 (coll. Nouvelle bibliothèque du Moyen Âge).
} 
(dans la Suite post-Vulgate) $^{27}$. Les continuateurs de Robert de Boron en font un révélateur des failles psychologiques du jeune Arthur, dans la mesure où leurs récits ne reproduisent pas le substrat structurel équilibré du prototype : les actes transgressifs de leur personnage ne sont pas tempérés par un personnage unifiant l'autorité spirituelle à l'autorité narrative. Sa défaillance s'explique donc par l'empire de passions qui le conduisent à engendrer une sorte de double négatif ${ }^{28}$ qui aggrave les failles morales initiales de son père.

L'épisode de la Fausse Guenièvre est, quant à lui, un cas complexe d'union entre une défaillance amoureuse et une perturbation profonde des facultés régaliennes et intellectuelles d'Arthur. Le roi a été fait prisonnier par les partisans de l'usurpatrice ${ }^{29}$, qui lui promet qu'elle ne le libérera pas avant d'obtenir la Table Ronde. Il s'éprend toutefois d'elle :

«Ensint remest li rois Artus o la dameisele que onques ses gens ne surent qu'il devint; et elle le venoit sovent veoir, tant que li rois la trova cortoise et de bones paroles, qui molt li plot, et que molt s'entroblia de l'amor qu'il avoit a la roine. Et tant com il demora en la prison, gisoit tos jors la damoisele avecques lui $»^{30}$.

Trois phénomènes président à la conversion amoureuse $\mathrm{du}$ roi : l'enfermement physique, la saturation concrète et intellectuelle de l'espace sensoriel par la Fausse Guenièvre et une révolution psychique fondée aussi bien sur l'alternance de menaces et de tendresse que sur une coupure absolue avec le

\footnotetext{
${ }^{27}$ Voir Robert de Boron, Merlin, édité par Alexandre Micha, Genève, Droz, 1979 (coll. TLF, 281), chapitres 52 à 65, p. 198-228.

28 Sur les dédoublements de personnages dans les Suites $d u$ Merlin, voir Christine FERLAMPIN-ACHER, « Le double dans la Suite du Roman de Merlin et la Suite Vulgate; faux frères, faussaires, féerie et fiction ", Jeunesse et genèse du roman arthurien, les suites romanesques $d u$ Merlin en prose, études réunies par Nathalie KoBle, Orléans, Paradigme, 2007 (coll. Medievalia), p. 33-52.

${ }^{29}$ Lancelot, op. cit, tome I (Genève, Droz, 1978, coll. TLF), chapitre VI, paragraphes 27 et 28, p. 102-103.

${ }^{30}$ Ibid., paragraphe 36, p. 107.
} 
milieu d'origine ${ }^{31}$. Le verbe entroublier souligne, quant à lui, un véritable bouleversement mélancolique : utilisé par la Première continuation de Perceval pour désigner une diversion mélancolique du roi, il fait partie d'un réseau lexical de l'oubli qui intervient à plusieurs reprises dans la description de ces états ${ }^{32}$. Sa valeur est donc nosologique, puisqu'il associe l'amour du roi pour la jeune femme à une pathologie du désir, mais aussi de la pensée ${ }^{33}$. Elle est également morale : en soulignant une crise du discernement et de la mémoire, le verbe met en évidence l'abolition de la conscience du personnage et disqualifie sa position intellectuelle. La défaillance est donc marquée aussi bien par le vocabulaire que par les symptômes d'une errance intellectuelle atrabilaire. Le récit oppose un amour fondé sur une aliénation du corps et de l'esprit à l'union matrimoniale et à l'amour courtois véritable ${ }^{34}$. Ces défaillances liées à l'amour confrontent l'être intime à l'être public, ou le «corps individuel» au «corps royal» ${ }^{35}$. Elles ne sont pas les seuls cas où Arthur fait défaut à sa vocation.

\section{Arthur et les crises de la souveraineté}

L'exemple des Prophesies de Merlin le montre : il n'est pas possible de séparer totalement le « corps intime » du « corps royal » quand l'on décrit les défaillances du roi Arthur. Il n'y a jamais d'antagonisme absolu entre lui et un

\footnotetext{
${ }^{31}$ Ibid., paragraphe 35, p. 106-107.

${ }^{32}$ Voir Chrétien De TROYes, Le Chevalier de la charrette, édité par Mario RoQues, Paris, Champion, 1958 (coll. CFMA), vers 715 (pour Lancelot); Le Chevalier au lion, édité par Mario Roques, Paris, Champion, 1967 (coll. CFMA), vers 52 (pour Arthur); Le Conte du Graal, op. cit., vers 4202 (pour Perceval).

${ }^{33}$ L'épisode pourrait donc illustrer la définition que donne Raison de l'amour dans la partie du Roman de la Rose écrite par Jean de Meun (voir l'édition de Félix LECOY, tome I, Paris, 1965 (coll. CFMA, 92), vers 4348 à 4354).

${ }^{34}$ L'amour qu'Arthur éprouve pour Camille conduit également à sa capture lors d'une guerre qui l'oppose aux envahisseurs saxons (Lancelot, op. cit, tome VIII, Genève, Droz, 1982 (coll. TLF), chapitre LXXa, paragraphes 32-35, pp. 441-443).

${ }^{35}$ Voir Ernest KantorowiCZ, Les Deux corps du roi : essai sur la théologie politique au Moyen Âge, Paris, Gallimard (coll. Bibliothèque des histoires), 1989 ( $1^{\text {ère }}$ éd. : Princeton, 1957).
} 
«mauvais roi ${ }^{36}$ ou des avatars de princes disqualifiés par leur nature « sauvage $»^{37}$ : les écrivains ne relatent pas la lutte d'un roi parfait contre des rivaux frappés de tares insurmontables. Arthur connaît quatre types de défaillances politiques : la vaillance chevaleresque et militaire, l'entretien de la cohésion de son réseau vassalique (ou de son lignage), l'exercice de la justice et les fonctions religieuses. L'envoi d'un chevalier incapable de remplir sa mission avec succès est un type important de défaillance chevaleresque. Dans le Perlesvaus, Arthur a rappelé auprès de lui Lancelot, qui a défendu victorieusement l'Écosse des attaques de Madaglan ${ }^{38}$. Il envoie à sa place Brian des Îles, devenu son sénéchal après la fuite de Keu, qui est défait par l'ennemi de son suzerain ${ }^{39}$. La défaillance est politique, dans la mesure où le roi désigne un ennemi qu'il a vaincu mais avec lequel il ne s'est pas formellement réconcilié ${ }^{40}$, et militaire : par son échec et par l'aversion qu'il éprouve envers Arthur, Brian des Îles devient l'allié de fait de Madaglan. La crise de la fonction royale passe donc par une politique contredisant les intérêts du royaume; elle va de pair avec un délitement du réseau vassalique, Lancelot étant arrêté et emprisonné sur le conseil de Brian ${ }^{41}$, qui a convaincu Arthur que les Écossais le porteraient sur le trône ${ }^{42}$. Les deux motifs du «sénéchal traître» et de l'arrestation du vassal le plus fidèle et le plus compétent structurent ici un bouleversement de la fonction royale motivé par l'effondrement des bases éthiques de la royauté d'Arthur et initié par la récompense que le roi accorde à Keu, meurtrier de son fils Lohot $^{43}$. Le roman décrit donc une logique de dissolution éthique insérée dans un mouvement narratif partant d'une crise

\footnotetext{
${ }^{36}$ Comme Marc dans le Tristan en Prose.

${ }^{37}$ Comme les rois saxons géants qui lui font la guerre dans l'Estoire de Merlin, ou le géant Rion du Roman de Tristan de Thomas.

${ }^{38}$ Le Haut livre du Graal, op. cit, branche X, p. 874.

${ }^{39}$ Ibid., p. 876.

${ }^{40}$ Ibid., p. $852-854$ et 876 .

${ }^{41}$ Ibid., p. 918-922.

${ }^{42}$ Ibid., p. 916.

${ }^{43}$ Ibid., branche VIII, p. 574.
} 
initiale pour décrire un sursaut moral suivi de l'effondrement éthique du monde arthurien. Les défaites des vassaux du roi contre Madaglan ${ }^{44}$ font le lien entre les défaillances d'Arthur et celles de ses chevaliers. Le Perlesvaus pousse très loin un principe de disqualification de la cour tout entière par la faute de son roi que l'on observe dans d'autres textes ${ }^{45}$, dans la mesure où il le fait reposer sur l'abandon et l'oubli de ses chevaliers au profit de son nouveau sénéchal ${ }^{46}$. Un conflit s'instaure entre des chevaliers positifs et un roi assimilé à des instances néfastes. Il réalise un risque qui existe ailleurs : la rupture de la cohésion du lignage. La jeunesse d'Arthur est, à cet égard, une période très critique. Le Perlesvaus et la rédaction V.I. du Tristan en Prose exceptés ${ }^{47}$, les autres textes de notre corpus la rendent problématique au début de son règne. Le Livre d'Artus évoque ainsi une très violente querelle l'ayant opposé à plusieurs de ses vassaux $^{48}$. La tension entre plusieurs niveaux de conscience extérieure et le comportement autonome du roi est caractéristique de ces moments où l'appui social du personnage est fragilisé ; elle sert à confronter la figure du souverain à une fonction référentielle qu'on attend qu'il satisfasse.

La fonction judiciaire est particulièrement propice à la confrontation de l'homme avec l'idéologie royale. Le roman de Jaufre ${ }^{49}$ montre qu'elle peut

\footnotetext{
${ }^{44}$ Ibid., branche X, p. 908.

${ }^{45}$ Voir Le Livre d'Artus, édité par H. O. SOMMER, The Vulgate Vrsion of the Athurian Rmances, tome VIII, Washington, Carnegie Institution, 1913, New-York, A.M.S Press, 1969), p. 151-153. Après le refus de Grex de tenter l'aventure de la Laide Semblance, plusieurs chevaliers d'Arthur y échouent à leur tour; la disqualification morale de la collectivité s'accompagne d'une mise en cause de sa valeur éthique et chevaleresque, plusieurs personnages tentant une épreuve réservée à Grex.

${ }^{46}$ Le Haut livre du Graal, op. cit., branche XI, p. 986.

${ }^{47}$ Voir le Tristan en Prose, V.I. (rédaction du manuscrit Paris, BNF fr. 757), tome I (édité par Joël Blanchard et Michel QuereuIL, Paris, Champion, 1997, coll. CFMA), chapitre VII, paragraphes 3-5, p. 325-331 : accompagné de Gaheriet et d'Hector des Marès, Tristan sauve de force les deux accusatrices d'Armant, condamnées par Arthur au bûcher ; Gauvain s'élance pour l'affronter.

${ }^{48}$ Le Livre d'Artus, op. cit., p. 14, lignes 31 à 33.

49 Édité par René LAVAUd et René NeLli, Les Troubadours, tome II : l'œuvre épique, Paris, Desclée de Brouwer, 2000, p. 17-618.
} 
entrer en conflit avec le désir de pardonner. Malgré sa colère ${ }^{50}$, Arthur pardonne à Taulat et prie Melian, qui l'a traduit devant lui, de faire de même ${ }^{51}$. Melian refuse de renoncer à lui demander de rendre des comptes pour les tortures qu'il lui a infligées ${ }^{52}$ :

«Que, seiner, anta corporal

Jamais a ome que ren val

Senz venjansa non l'ieis del cor,

Mais a malvatz oblida e mor.

Per qu'ie.us prec, si.us plas, jujament

Que.1 me fassas far leialment

De l'amta c'a mon corz a faita, C'anc mais sa par non er retraita $»{ }^{53}$.

La magnanimité s'oppose à l'impératif de justice; le récit confronte la résolution instinctive d'un conflit initial entre Taulat et le roi à des procédures « littéraires » et juridiques qui ont une fonction civilisatrice : elles médiatisent l'antagonisme en permettant à l'aristocratie chevaleresque d'assumer une fonction régulatrice. Arthur renonce à un impératif judiciaire destiné à combattre le monopole de la subjectivité passionnelle et émotive ${ }^{54}$ qu'il veut réintroduire en pardonnant, alors même que la reconnaissance de ses prérogatives par Jaufre suppose implicitement qu'il les exerce ${ }^{55}$. La scène confronte un garant de la moralité chevaleresque et des cadres coutumiers à des instances favorables au

\footnotetext{
${ }^{50}$ Voir les vers 6461-6465.

${ }^{51}$ Voir les vers 6577-6593.

${ }^{52}$ Voir les vers 6594-6623.

${ }^{53}$ Vers 6623-6630 : «Parce que, seigneur, la honte infligée au corps ne sort jamais du cœur d'un homme qui vaille quoi que ce soit sans qu'il ne s'en soit vengé. Chez les vils, par contre, elle est oubliée et s'éteint. C'est pourquoi je vous prie, s'il vous plait, de faire prononcer pour moi un jugement loyal de l'outrage qu'il m'a fait, dont jamais on ne racontera son pareil ».

${ }^{54}$ Voir Pierre LEVRON, «Mélancolie, émotion et vocabulaire : enquête sur le réseau lexical de l'émotivité atrabilaire dans quelques textes littéraires des douzièmes et treizièmes siècles ", à paraître dans les actes des journées d'études E.M.M.A (Les émotions au Moyen Âge), sous la direction de Damien BoQueT et de Piroska NAGY.

${ }^{55}$ Jaufre, op. cit, voir les vers 6539-6542.
} 
pardon et à l'oubli, le roi et son sénéchal ${ }^{56}$. La rédaction V.I. du Tristan en Prose décrit l'incapacité d'Arthur de garantir le «droit fondamental ». La défaillance judiciaire du roi se fonde sur la tension entre l'exactitude d'un processus judiciaire apparent et la réalité : il n'a pas vu la traîtrise de Marc au cours du combat qui l'oppose à Armant. Il devient donc le garant d'une erreur judiciaire d'autant plus subversive pour la société arthurienne que sa cour est un exemple de rigueur procédurale ${ }^{57}$. Les défaillances liées à l'exercice du droit coutumier sapent les bases idéologiques de la royauté institutionnelle en se fondant sur des conflits essentiels entre les principes implicites ou explicites de cette dernière et les faiblesses de l'homme qui en est investi, mais l'échec judiciaire d'Arthur fragilise davantage le travail de civilisation qu'il entreprend ou qu'il doit entretenir que les autres défaillances sociales ou politiques, dès lors qu'il intervient toujours au moment où sa position autoritaire est la mieux assise ${ }^{58}$ : les personnages de Jaufre et du Tristan en Prose font appel à lui parce qu'il est une référence morale et judiciaire dont l'on propose spontanément l'intervention ${ }^{59}$.

Plus préoccupantes pour une mentalité médiévale sont les défaillances religieuses du roi. Dans le Lancelot en Prose, un religieux explique à Arthur que son risque de perdre la guerre que Galehaut mène contre lui est lié de près à une damnation menaçante ${ }^{60}$. Se fondant sur une définition implicite d'un roi-juge (évoquée par les deux références insistantes à leur «droit») dont l'âme doit inspirer le jugement, assez comparable à celle que l'on rencontrera quelques décennies plus tard chez Brunetto Latini ${ }^{61}$, le religieux met en évidence une

\footnotetext{
${ }^{56}$ Voir les vers 6631-6636.

57 Tristan en Prose, V.I., tome I, op. cit., chapitre VII, paragraphes 1-2, p. 324.

${ }^{58}$ Il s'oppose donc à sa fragilité politique et aux risques d'instabilité de son lignage et de son réseau vassalique en déplaçant la question centrale de la légitimité du souverain de ses origines vers sa pratique.

${ }^{59}$ Voir le Tristan en Prose, V.II., tome IV, op. cit., chapitre II, paragraphe 10, p. 74-75.

${ }^{60}$ Lancelot, tome VIII, op. cit., chapitre XLIXa, paragraphe 18, p. 13-14.

${ }^{61}$ Voir Li Livres dou tresor, édité par Francis J. CARMODY, Berkeley, 1948, Genève, Slatkine Reprints, 1998, livre II, éthique V, paragraphe XXVIIII, p. 199.
} 
sorte d'acédie politico-religieuse caractérisée par la négligence du roi. L'épisode de la Fausse Guenièvre, quant à lui, met plus clairement en cause la fonction royale de modèle social et religieux. Arthur a refusé de se réconcilier avec la vraie Guenièvre, qu'il a confiée à Galehaut ${ }^{62}$; son union avec l'usurpatrice interrompt la vie religieuse dans son royaume ${ }^{63}$. Mesure exceptionnelle dans la matière de Bretagne, l'interdit jeté sur le Logres s'apparente à une déchristianisation punitive d'un royaume où les commandements ainsi que le sacrement du mariage sont enfreints par le souverain. Le divorce de fait du couple royal est quant à lui une faute théologique essentielle. L'intervention du pape étend les conséquences des amours irrégulières du roi avec l'usurpatrice à l'ensemble du corps social. Substitut humain de la colère divine, celle du souverain pontife se fonde sur une exigence d'exemplarité qui conduit à la dissolution de l'autorité spirituelle d'un roi qui doit là encore garantir le respect de la loi divine dans son royaume; elle précède la maladie qui s'empare d'Arthur $^{64}$, de la Fausse Guenièvre et de Bertholay, son conseiller ${ }^{65}$, et qui est, quant à elle, une sanction divine. Privés de légitimation théologique, les actes politiques inspirés par des passions individuelles sont foncièrement injustes.

Les défaillances d'Arthur jouent un rôle important dans la constitution d'une royauté mythique, parce qu'elles confrontent un être humain à des impératifs sociaux mais aussi psychologiques et spirituels qui vont bien au-delà de sa dimension microcosmique. Elles permettent de mesurer les limites des capacités humaines en tenant compte de la faiblesse des hommes aussi bien que de leurs possibilités. Leur apport au mythe littéraire du roi Arthur est également métaphorique: image idéal du souverain, le personnage attire une certaine identification de la part des destinataires de la matière de Bretagne, tout comme

\footnotetext{
${ }^{62}$ Lancelot, tome I, op. cit., chapitre VIII, paragraphes 48 à 52, p. 145-147.

${ }^{63} \mathrm{Ibid}$, chapitre IX, paragraphe 4, p. 153.

${ }^{64}$ Ibid., paragraphe 10, p. 156.

${ }^{65}$ Ibid., paragraphes 5 et 6 , p. 153-154.
} 
d'autres héros mythiques. Elles permettent alors de parler des faiblesses ou des défaillances des hommes réels. En plaisant et en instruisant, la littérature utilise une fonction anthropologique importante du récit mythique... 